\title{
La producción del espacio urbano: Entre materialidad y subjetividad Daniel Hiernaux Nicolas
}

Prof.Inves, Universidad Autónoma de Querétaro, SNI III, correo: danielhiernaux@gmail.com Recibido: 25-10-18 Aceptado: 12-01-2019 Publicado: 15-08-2019

Cómo citar este artículo: Hiernaux D. (2019) "La producción del espacio urbano: Entre materialidad y subjetividad" en Revista Científica de Estudios Urbano Regionales Hatsö-Hnini, Año 1, Núm. 1, Abril-Septiembre 2019, págs. 1-14 México DOI: https://doi.org/10.47386/2019V1N1AE1

\section{INTRODUCCIÓN}

La "producción del espacio" urbano se ha vuelto una expresión ampliamente aceptada por todos los medios sean académico, profesional o político. Todavía en los setenta, el concepto pertenecía al vocabulario de cuño marxista que se había impuesto en los estudios urbanos, particularmente a raíz de los trabajos de los autores de la llamada sociología francesa urbana crítica, liderada por Manuel Castells, Edmond Preteceille, Christian Topalov entre otros.

Sin embargo, quien le dio fuerza al concepto de producción del espacio (urbano o no) fue indudablemente Henri Lefebvre con su magna obra La production de l'espace publicada en 1974 en francés, mucho más tardíamente en inglés en 1999 e inexplicablemente solo recientemente en castellano (Lefebvre, 2013). Obra críptica por excelencia, "La producción del espacio" tuvo entre otros el mérito de analizar las prácticas sociales como actos de producción, en el sentido de una transformación de una "materia prima" aplicando una "fuerza de trabajo" que genera un producto de un valor mayor es decir con una "plusvalía".

Obvio que esta forma de plantear la producción del espacio es consecuencia lógica de los trabajos de Carlos Marx sobre la transformación del mundo material a partir de la integración del trabajo al insumo material, para lograr, como resultado, un objeto con un valor final superior al valor de todas las componentes integradas al mismo.
Plusvalía, valor, capital, fuerza de trabajo y otros, todos estos conceptos de cuño marxista se integraron entonces en una panoplia conceptual que permitió pensar el espacio de manera distinta de por el pasado: el espacio considerado en adelante como un producto de la sociedad.

Obvio que esta forma de plantear la producción del espacio es consecuencia lógica de los trabajos de Carlos Marx sobre la transformación del mundo material a partir de la integración del trabajo al insumo material, para lograr, como resultado, un objeto con un valor final superior al valor de todas las componentes integradas al mismo. Plusvalía, valor, capital, fuerza de trabajo y otros, todos estos conceptos de cuño marxista se integraron entonces en una panoplia conceptual que permitió pensar el espacio de manera distinta de por el pasado: el espacio considerado en adelante como un producto de la sociedad.

Lo anterior no se hizo sin omitir unos debates o por lo menos tomar partido con relación a los mismos, sobre el significado mismo de espacio. Bien se sabe que la filosofía y la geografía han contribuido de manera sustancial a tratar de esclarecer los posibles significados del espacio (Hiernaux y Lindón, 1993): más aún, si se asume que el espacio es realmente existente (no es una opinión compartida por todos los filósofos) entonces es posible discutir las distintas formas de producción del mismo y los resultados que se pueden esperar. Es entendible que las ciencias sociales de los 60 en adelante, ávidas de pensar la relación sociedad-espacio que había sido tan menospreciada durante las décadas inmediatamente anteriores, aceptarían con cierta facilidad estos nuevos planteamientos. 
Pero esta asimilación de las transformaciones del espacio a una producción del mismo no avanzó y todavía no avanza sin problemas: podemos anticipar ya la idea que se desarrollará posteriormente en el presente trabajo, es que se gestó de esta manera una concepción materialista del espacio, supuestamente teñida de objetividad, que acabó por "reificar" el espacio sea urbano o no. Esto es lo que se desarrollará en las páginas que siguen. En una primera parte, se analizará a detalle lo que significa "producir el espacio" desde la concepción tradicional de origen lefebvriana de la expresión. En un segundo tiempo, se expondrán las limitantes de esta óptica para introducir la necesidad de una ampliación del sentido mismo del concepto de "producción del espacio" para integrar las dimensiones no materiales del espacio y de la relación de la sociedad con el mismo.

\section{La producción material del espacio urbano}

El planteamiento sobre la producción material del espacio establece, implícitamente, una concepción particular del espacio: éste está entonces formado por una serie de objetos con una localización determinada y una ocupación de un área espacial dependiendo de su forma y sus medidas, así como del espacio entre los mismos objetos.

Producir el espacio implica entonces actuar sobre ambas componentes: los objetos y el espaciamiento entre otros.

Por ello, producir el espacio puede referirse tanto a la edificación, transformación / adecuación o misma demolición de ciertos objetos en el espacio, como a intervenir sobre la configuración determinada por la posición recíproca de los objetos, la forma y el espacio ocupado por los mismos, así como por el espaciamiento entre ellos. En este mismo tenor, la producción del espacio se diferencia singularmente de la producción de un objeto industrial, por ejemplo. En efecto, la producción del objeto trabaja directamente sobre la materia prima aplicando fuerza de trabajo e instrumentos de trabajo de de manera a transformar ese conjunto en un objeto que adquiere por lo mismo un valor de uso y un valor de cambio. Es en ese proceso que se genera entonces la plusvalía. La producción del objeto artesanal o industrial trabaja entonces sobre un objeto, pero no sobre la configuración de un conjunto de objetos que es también parte del proceso de producción del espacio.

De tal suerte, la producción del espacio desencadena una transformación más amplia: remite no sólo a transformar la materia prima en un objeto o un objeto en otro, sino a trabajar sobre el espaciamiento y la disposición de estos en la trama espacial subyacente a ellos.

Por ello es que un objeto urbano producido y dispuesto en un lugar específico, no adquiere el mismo valor según su posición en el entramado espacial y su relación con los demás objetos. Tomemos un ejemplo: producir un punto de venta y consumo de café, no tendrá el mismo valor si se ubica en una colonia o en otra. La diferencia provendrá, como bien se hizo notar desde los sesenta, de las rentas diferenciales que se generan en una localización u otra, pero también de otros factores que descansan en el resultado de la configuración de los espacios. 
Por ejemplo, fábricas integradas a un cluster especializado probablemente lograrán producir mayores valores de cambio que si son sólo empresas ubicadas de manera independiente del entorno. Lo anterior, trabajado por varios autores en los sesenta y setenta, conduce a percibir que en el proceso de producción del espacio se va a encontrar tanto producción de plusvalor como producción de rentas diferenciales.

Aplicado a la ciudad, la producción del espacio da por resultado, en primer lugar, lo que Emilio Pradilla ha llamado "los soportes materiales" de lo urbano (Pradilla, 1984). Por ello entiende los productos específicos que hacen la ciudad desde sus viviendas, sus redes de servicio o sus cárceles, por ejemplo. Si bien la taxonomía resultante de repasar el conjunto de "objetos urbanos" es interesante, no es menos cierto que la dimensión morfológica no es tomada en cuenta desde esa aproximación. En otros términos, la producción de la ciudad se vuelve restringida a una definición desde objetos urbanos, sin considerar la morfología del espacio como resultado de la disposición de los mismas en el espacio y las relaciones potenciales entre sí.

Y, por otra parte, es evidente que esta disposición es central para entender la ciudad como producto: la ausencia de ciertos objetos urbanos en algunas partes de la ciudad, como un hospital o una escuela, contribuye a una diferenciación entre los ocupantes de tal o tal barrio, y por ende una posible segregación sociourbana.
También, desde la lógica del capital, Lefebvre nota que: "es en el espacio y por el espacio donde se produce la reproducción de las relaciones sociales de producción capitalista. El espacio deviene cada vez más en un espacio instrumental" (Lefebvre, 1974b: 223).

La toma en cuenta de la morfología como resultado también de un proceso de producción de la materialidad urbana, lleva a reflexionar nuevamente sobre la conceptualización del espacio: Bien se sabe que se ha insistido en que se han desarrollado tres concepciones del espacio.

La primera, el espacio "continente" o contenedor, cuya función principal es contener los objetos urbanos y las personas; la segunda por la cual el espacio es reflejo de la sociedad que lo ocupa; ésta es la más socorrida por los investigadores marxistas de la ciudad, al considerar que leer la ciudad conduce a poder reconocer, por ejemplo, las desigualdades sociales, las posiciones diferenciales de las clases sociales en el espacio, el despla-zamiento de una clase por otra en un espacio (entre otros por "desposesión" como lo plantea David Harvey); la tercera, que de lejos es la más interesante, piensa al espacio como una configuración que interactúa con la sociedad, es decir que se establece una relación en ambos sentidos: de la sociedad hacia el espacio, y del espacio hacia la sociedad (Hiernaux y Lindón, 1993), modelándose mutuamente. 
Desde una perspectiva analítica que se interesa solamente en los objetos como soporte de la actividad humana, entonces estamos claramente en el caso de una conceptualización del espacio como contenedor. A lo más, como lo señalamos en el párrafo anterior, en los textos de cuño marxista más convincentes, se apreciará una conceptualización del espacio como reflejo de esa sociedad. Pero nosotros apuntamos a plantear que el espacio urbano, producto de este proceso de producción, interactúa claramente con la sociedad de tal forma que se puede afirmar que el espacio es a su vez producto y productor de la sociedad. Pensado así, no hay posibilidad de que la configuración en sí no sea relevante para el análisis de la relación sociedad-espacio $y$, por ende, que no sea resultado de un proceso de producción del espacio.

Tomando un ejemplo extraído de la vida cotidiana, podemos ver que los habitantes de una casa no solo producen el espacio de su cotidianidad a partir de la compra y ubicación de muebles en tanto objetos en el espacio, sino que la disposición que de ellos realizan también tiene sentido y es producida por los habitantes además de ser muchas veces relevante para la manera como ocupan el espacio. Podría ejemplificarse eso a través de los casos en los cuales los habitantes de un cuarto redondo suelen colgar ciertos utensilios y arrinconar los muebles en la noche para disponer del espacio para dormir.
La producción de la disposición de los objetos se vuelve entonces una estrategia central para aprovechar un espacio de vida muy reducido y lograr ejercer todas las funciones necesarias a pesar de su limitada superficie.

\section{"espacio como una configuración que interactúa con la sociedad"}

Asimismo, la construcción de casas individuales bajo una disposición de fraccionamiento tradicional es muy distinta de un conjunto producido bajo las normas del New Urbanism, por el cual ciertos espacios se dedican prioritariamente a una potencial vida más colectiva. Esto sin prejuiciar que se logre efectivamente. En este ejemplo, podemos encontrar claramente la presencia de una estrategia de instrumentalización del espacio para fines de ganancias o promoción del producto.

Estamos entonces frente a un proceso de producción del espacio de doble cara: producción de objetos y producción de morfología. Asimismo, 
se puede asumir que la morfología en sí, producto de una sociedad, y los objetos urbanos creados no son solo reflejos de la sociedad, sino una componente medular de la vida social que, hasta cierto punto, determina las prácticas sociales que se realizan en ese espacio particular.

La pregunta que viene después es si ¿esta producción material del espacio recubre todas las formas posibles de producción del espacio? Obviamente que no, y por ello es necesario de extender el concepto de producción del espacio a dimensiones no materiales.

\section{La producción subjetiva del espacio urbano}

Los diversos giros que han conocido las ciencias sociales son hoy bien conocidos de todos: giro lingüístico, giro hermenéutico, giro cultural, giro biográfico, son algunos de los principales. Aplicados al espacio, particularmente desde la geografía humana, han permitido poner en tela de juicio "...las categorías y conceptos geográficos de los cuales nos valemos para descifrar las variadas y complejas relaciones de la sociedad con el espacio" (Lindón, 2010: 23). Estos giros los podemos ver actuar tanto dentro de la esfera de lo conceptualteórico como de aquella que remite a la metodología.

En este tenor, Ángelo Turco, destacado geógrafo italiano, plantea que la geografía occidental ha recurrido a dos modelos de representación espacial: el primero es el espacio paratáctico, el segundo el espacio liminar.
El espacio paratáctico, plantea el autor, "...tiene que ver con las secuencias,

localizaciones, coorden adas $y$ cosificaciones de las características naturales o antrópicas de la superficie terrestre" (Turco, 2010: 92). Esa figura narrativa del espacio ha permitido construir modelos sintácticos del espacio que ofrecen alg u $n$ as explicaciones de la estructuración misma del espacio y plantean, en ciertos casos, la posibilidad de desarrollar modelos predictivos.

Por su parte la figura narrativa del espacio liminar se asocia con formas es paciales que remiten a 1a indeterminación, la imprevisibilidad de lo que el humano realiza y produce. Como bien se pueden deducir, el análisis de la producción del espacio desde la materialidad del mismo, es decir aquella que genera objetos, espaciamiento y configuraciones se nutre de la figura narrativa paratáctica. En ese campo, podemos ubicar los modelos y los análisis causales de la geografía tradicional en particular de aquella que apostó a la definición del espacio como plano euclidiano y a su estudio como un producto del análisis cuantitativo. Remite entonces a esa corriente apodada "geografía teorética-cuantitativista". De la misma manera, el hecho de que no todo está cubierto por esa tendencia, por ejemplo, la relación de doble vía del espacio a la sociedad y de la sociedad al espacio, nos permite inducir que la figura narrativa del espacio liminar no puede ser eludida y se convierte, desde nuestra perspectiva, en la que centralmente otorga sentido al análisis del espacio. 


\section{La producción del espacio urbano: Entre materialidad y subjetividad}

Así Turco afirma contundentemente que estamos en una etapa de "crisis del espacio paratáctico y de retorno del espacio liminar" (Turco, 2010: 106). Si asumimos esta posición, entonces debemos elaborar una nueva propuesta con relación a la producción del espacio que es lo que se desarrolla de manera aun preliminar en esta segunda parte del ensayo.

Esta propuesta parte de un presupuesto inicial: La necesidad de rebasar la "reificación" como tratamiento instrumental de las cosas y las personas y por ende también del espacio urbano y del uso que hacemos del mismo. El concepto de "reificación" propuesto por Georg Lukács (1923) remite a una suerte de colonización del mundo vivido por la generalización unidimensional del intercambio mercantil a toda interacción soc i a l. Ella implica u n "desprendimiento" que significa que la persona ya no está afectada emocionalmente por los eventos o las personas. En otros términos, la reificación plantea la sola existencia de las reglas del juego que parten de la materialidad como sustento de la vida social, exentas de cualquier sentimiento o emoción, por lo que los actores, de esta manera, solo se aferran a las dimensiones cuantitativas y materiales de las cosas, los procesos y las personas. Habrá que recordar que Walter Benjamín sometió su análisis de la premodernidad en los pasajes cubiertos parisinos a una concepción similar por la cu al considerada que el mismo ser humano se reificaba progresivamente con el avance de la modernidad. Véase por ejemplo, la figura de la prostituta, "...imagen de la forma mercancía..." (Buck Morss, 1995: 253; Benjamin, 2005: 491-514).
Por su parte, Axel Honneth plantea que la reificación es una tendencia a olvidar que antes del conocimiento está el reconocimiento (Honneth, 2005): no podemos aprehender el mundo material si no es a partir del reconocimiento de la manera como otras personas lo han reconocido antes que nosotros. Recordando el célebre aforismo de Teodoro Adorno en Minima Moralia (Adorno, 2004) según el cual "un ser humano se vuelve realmente humano imitando otros seres humanos", Honneth plantea que nuestra aprehensión del mundo material pasa por la relación con otros seres que han hecho este proceso antes que nosotros y que, de esta manera, nos transmiten su experiencia del mundo. Así entonces entendemos que nuestra apropiación (y posterior producción) del mundo material solo puede darse a partir de una experiencia compartida, la cual no puede ser más que subjetiva.

De esta manera queda claro que la producción del espacio no es solamente el proceso de transformar la materia, colocar y organizar espacialmente el espacio, sino un proceso mucho más complejo por el cual se produce colectiva y subjetivamente el mundo que nos rodea.

Entramos así en el territorio tan complejo de la subjetividad aplicada al espacio, y por ende a una figura narrativa muy distinta de la que se ha aplicado en los análisis tradicionales del e s p a cio y d e s u p r o d u c - ción, donde prima el objeto y la materialidad del espacio en general: estamos entonces bien anclados en el campo del espacio liminar tal y como lo define Turco. 


\section{La producción del espacio urbano: Entre materialidad y subjetividad}

De hecho la expresión "bien anclados" es hasta cierto punto incorrecta: pisar el espacio liminar como figura narrativa implica desprenderse de las seguridades, de las certezas y de todos los referentes tradicionales que nos hacían ver el mundo de una manera particular y falsamente transparente. Nos introducimos, como exploradores, en un mundo hecho de incertidumbres, de complejidad, y de recorridos laberínticos, los cuales podemos dudar que conduzcan alguna vez a una salida única. Quizás habría que pensar que son tan variadas las salidas, que el verdadero avance es ser capaz de recorrer la figura laberíntica en vez de solo seguir la vía recta y aburrida de la autopista de la materialidad.

¿Cómo entonces redefinir la producción del espacio en el marco de la figura narrativa del espacio liminar? Se harán a continuación una serie de observaciones relacionadas con esa producción, sus actores y sus dimensiones, para tratar de reconstruir así el concepto mismo de "producción del espacio" con un sentido más amplio que articula la materialidad y la subjetividad.

En primer lugar, la producción del espacio implica siempre una experiencia previa del espacio, la cual, siguiendo a Honneth, debe partir del reconocimiento de la experiencia del otro. Como bien se supone, esta posibilidad del reconocimiento se vuelve cada vez más difícil en un mundo donde la individualización es creciente. Incapaces de reconocer no solamente el otro sino peor aún la experiencia del otro por el mismo desprendimiento[1], nuestra

[1] Habrá que recordar también el análisis de Georg Simmel del desprendimiento del hombre moderno frente a la ciudad y a sus demás habitantes, como anomia resultante del exceso de estímulos que genera la vida urbana. producción del espacio entonces tiene todas las condiciones para adscribirse a la reificación que comentamos anteriormente. Pero eso es solo parcial. Es evidente que la producción del espacio se mantiene como una práctica social compleja y podríamos llamarla también "poliactoral", es decir sometida a la intervención de múltiples actores. Aun cuando realizo un acto individual como construir mi casa, produzco al espacio de una manera colectiva, porque me integro al saber colectivo de la sociedad que me precede y que dejó huellas en mi predado de que tengo que realizar este acto de producción según ciertas normas que responden a la experiencia adquirida por los que me precedieron.

Si la experiencia precede entonces al conocimiento, no puedo evitar de darme cuenta que introduzco elementos subjetivos en el proceso de producción del espacio. Lo subjetivo puede provenir de diversas dimensiones, articuladas entre sí. Por ejemplo, el hecho de seguir ciertos ritos de fundación para las ciudades, o ritos de fertilidad para la casa recién construida. También en los modelos constructivos, se apuesta a reconocer ciertos patrones que fueron instituidos por el pasado y que han hecho sus pruebas. En ello, no interviene tanto la objetividad que quizás permitiría pensar en rectificar ciertos aspectos del modelo y además guiar hacia una innovación, sino la asimilación sin discusión de la validez de los modelos anteriores. Esto me ha parecido particularmente importante cuando estudié la periferia de la ciudad de México, observando como las zonas de desarrollo informal asumían ciertos 


\section{La producción del espacio urbano: Entre materialidad y subjetividad}

patrones de urbanización que no les era impuesto toda vez que actuaban fuera la normatividad instituida: así, los nuevos fraccionamientos informales siguen los patrones de damero que se han impuesto en las ciudades y que también rigen en las urbanizaciones empresariales. Lo anterior resuelve por cierto, aunque sea de manera parcial, la inquietud que surge en las investigaciones cualitativas sobre la representatividad de los casos estudiados: en cada individuo florece la experiencia colectiva, por lo que el interesarse en su experiencia individual es una forma de reflejar procesos societarios de mayor alcance. Es, en otros términos y con una lógica analítica distinta pero acorde, lo mismo que se plantean autores que reflexionan sobre los hologramas sociales (Lindón, 2007; Navarro, 1994).

Una segunda manera de introducir la dimensión subjetiva en el entendimiento de los procesos de producción del espacio urbano, es interrogarse sobre los imaginarios. Como lo hemos demostrado colectivamente en un libro reciente (Lindón y Hiernaux, 2012), los imaginarios son centrales para entender los procesos sociales y, por lo que nos concierne, los espaciales. Los imaginarios los hemos podido considerar como "actantes" es decir como elementos que usamos para producir el espacio (Hiernaux, 2007). En ese sentido, tanto como la fuerza de trabajo o las materias primas, los imaginarios se integran al proceso de producción de valor a través de las transformaciones del espacio urbano. Podemos así entender el papel tan significativo que logran tener los imaginarios en la producción de nuestro mundo de vida.
Para el empresario productor de espacios comercializables, el manejo de los imaginarios no es diferente del manejo de un trascabo; el empresario instrumentaliza los imaginarios, los vuelve herramienta con la cual trabaja el espacio, lo cubre así de sentido y alcanza su meta: hacer atractivo y vendible este espacio para su mercado objetivo.

Estudiar los imaginarios del miedo, por ejemplo, nos permite observar a qué grado las construcciones actuales pero también la morfología de los barrios (por ejemplo en su dimensión abierta/cerrada) refleja miedos más subjetivos que objetivos que recorren nuestras sociedades como figuras fantasmagóricas. Es a través del estudio del imaginario que podremos entender esa tendencia no solo a cerrar sino a blindar la casa, de la misma forma que las autoridades mexicanas suelen hablar de "blindar" la ciudad o la demarcación territorial de un subdivisón territorial para prevenir la violencia. Los imaginarios no son entonces el producto de la imaginación considerada por mucho tiempo como la "loca de la casa", sino herramientas de producción del espacio (entre otras finalidades). De tal manera, también se puede entender que es posible manipular los imaginarios, tal y como se manipulan las herramientas para lograr ciertos fines: ello es la "imagineria" o la "ingeniería imaginal" de la cual usan y abusan los medios publicitarios para convencernos de ciertas acciones, entre las cuales, comprar cierto tipo de vivienda en localizaciones determinadas. 


\section{La producción del espacio urbano: Entre materialidad y subjetividad}

Una tercera dimensión al estudio de la subjetividad en la producción del espacio, es el reconocimiento de que el acto de trabajo que lleva a la producción de la ciudad, es un acto que no solo produce material sino también simbólicamente la ciudad. El reconocimiento de la producción simbólica se ha hecho desde tiempo atrás por los antropólogos entre otros (Hannerz, 1986) y por quienes se adhieren a los estudios culturales. Sin embargo, no se ha examinado suficientemente ese proceso de producción simbólica del espacio desmenuzando sus componentes. Vale observar que la producción simbólica tiene por lo menos dos lecturas: la producción que usa símbolos para producir y otorgar valor a ciertos objetos y configuraciones urbanos, y la producción de símbolos urbanos en sí, sea objetos- símbolos, sean símbolos inmateriales; los cuales son dos procesos muy diferentes entre sí. La producción que usa símbolos para producir y otorgar valor es justamente una producción compleja que no solo usa elementos materiales para producir el espacio. Es aquella que usa al mismo tiempo o por lo menos de manera concurrente en el proceso de producción, materialidad y subjetividad. Usa el símbolo como un insumo para lograr ciertos fines, traducidos en ese caso en ciertos objetos o configuraciones susceptibles de ser usados de la manera deseada por el productor de este espacio. Por su parte, cierta producción del espacio manufactura símbolos tanto como objetos materiales recubiertos de un valor simbólico particular, como símbolos inmateriales.
A manera de ejemplo, para el primer caso podemos pensar en la producción de Arcos de Triunfo para celebrar un soberano, de monumentos para reflejar la memoria de los caídos en la guerra o las víctimas de un genocidio o de un holocausto. En México, la muy mentada Estela de Luz cabe en esa categoría, además de correr el riesgo de simbolizar en el futuro la máxima corrupción del gobierno calderonista. En todos esos casos, el símbolo está apegado directamente a la materialidad de la obra, en otros términos, se produce la obra material al mismo tiempo que el símbolo, jugando con el emplazamiento, la integración al contexto urbano, la forma, los colores y acabados, etc. La producción de símbolos inmateriales es, con toda evidencia, mucho más difícil de detectar. Remite a una capacidad de ver lo que no es visible: es decir captar una dimensión simbólica que no es materializada en una obra o una configuración espacial determinada. La actuación de un cilindrero por ejemplo, produciendo paisaje musical y por ende produciendo espacio a secas, actúa como un símbolo de la persistencia de tradiciones en una ciudad. Remite a tiempos pretéritos, a tiempos perdidos en un mundo que parecería solo valorar el instante presente al haber perdido la consistencia, el espesor del tiempo histórico. El referente simbólico no está situado en la persona, sus instrumentos o su vestimenta, pero más bien en la recreación mental que puede hacerse de un paísaje urbano desaparecido del cual él es un sobreviviente persistente y casi solitario. 


\section{La producción del espacio urbano: Entre materialidad y subjetividad}

La geografía humana reciente, o por lo menos cierta geografía que ha asumido por propia la figura narrativa del espacio liminar, se ha puesto a investigar estas dimensiones colectivas, imaginarias, simbólicas de la producción y de la apropiación del espacio urbano. Y es en ese sentido que surge una duda: ¿puede disociarse la producción del espacio urbano de su apropiación?

Todo lo anterior indicaría lo contrario: la apropiación del espacio urbano, es parte del proceso de producción del mismo. Esto difiere por supuesto de la conceptualización anterior de la producción del espacio que plantea en cierta forma que el uso del espacio - su "consumo"- debe distinguirse de la fase meramente productiva del mismo. Lo anterior sitúa entonces la producción del espacio como un tiempo, un momento de la intervención del espacio, seguido por otra fase de consumo. Obvio que frente a lo anteriormente dicho, esta fragmentación en dos tiempos consecutivos (producción y posterior consumo) es perfectamente contradictoria.

El espacio es producido toda vez que se le aplica transformaciones tanto materiales como inmateriales. Por ello, ocupar el espacio, desarrollar prácticas espaciales de manera individual (aun con contexto grupal -por reconocimientocomo lo señala Honneth) y colectiva es una forma de producción del mismo. Estamos así evitando la dicotomía tan tradicional entre productores y usuarios del espacio urbano, una herencia nefasta de los enfoques economicistas en los estudios urbanos, dicotomía particularmente impropia en nuestro ver, porque implica subjetivamente que los productores son los que tienen el poder, y los usuarios los que "padecen" el espacio producido por los anteriores. No podemos poner en duda que ciertas configuraciones del espacio y ciertos espacios-objetos particulares son padecidos por los ocupantes, pero eso no implica que toda práctica socioespacial -del espacio y en el espacio-se encuentra forzosamente sometida al diktat de supuestos productores que mantienen el poder.

Más aun, es evidente que no todos los actos de producción del espacio son intencionales: ciertas configuraciones espaciales - particularmente en los espacios públicos urbanos - pueden ser subvertidas por los usuarios que producen así un espacio "otro", radicalmente diferente en su materialidad y/o su subjetividad. Plazas que se reconvierten en espacios ocupados por disidentes (por ejemplo los Okupas), y se tornan así en espacios símbolicos de una resistencia. La mejor prueba de ello es cuando las misma autoridades se molestan y desocupan el lugar, porque "no se respecta" el uso atribuido al espacio de referencia por las "autoridades competentes".

Apropiarse del espacio es entonces producirlo tanto como un objeto que transformamos en su materialidad y en su subjetividad. Quisiera traer a colación un ejemplo de las prácticas socioespaciales en la muy conocida y remozada calle de Regina en el Centro Histórico de la ciudad de México, espacio predilecto del gobierno de la ciudad y de aquellos que ejecutan las políticas empresariales de Carlos Slim sobre algunas porciones de la capital mexicana. 


\section{La producción del espacio urbano: Entre materialidad y subjetividad}

Se observa cómo una calle lateral a Regina fue dotada de árboles en macetas y de bancas que generan un ambiente más de detenimiento que de circulación que es a lo que invita la remodelación de Regina. Pero ese espacio se usa y por ende se produce incesantemente por quienes lo ocupan, desde vecinos, turistas y paseantes o trabajadores de limpieza. Este acto, no es apropiación, es literalmente una nueva producción de un espacio para fines propios, subvirtiendo el uso mismo que se había previsto para ese rincón de la ciudad. Por lo mismo, producen un espacio propio, aun si es de carácter efímero.

Otro ejemplo es el uso de la plaza Zarco, situada cerca de la Iglesia de San Hipólito en el centro de la capital mexicana. Estudiado por Paulina Mejía (2012), este espacio público es producido literalmente cada día 28 de cada mes y en particular el 28 de octubre cuando se realizan las procesiones al supuestamente milagroso San Judas Tadeo, santo patrón de las causas difíciles. En esas ocasiones la asistencia es tanto de devotos de todas las clases sociales que acuden a la iglesia y a su atrio para venerar su santo preferido, como también por la tribu juvenil de los "Reggaetoneros" que realizan ciertos ritos cristianos pero que sobretodo producen el espacio de la plaza Zarco para su fiesta particular, con sendas bebidas y drogas, dentro de ritos tribales que les son propios.

Limitar el análisis de estos dos casos a una simple apropiación de un espacio producido ex ante sería un notable error. En ambos casos se asiste a una producción del espacio, tan interesante como la producción de enormes fraccionamientos nuevos de muchas viviendas.
En la vida cotidiana, producimos el espacio de manera incesante; no siempre tenemos acceso a la perennidad de la construcción de las catedrales medievales o de las pirámides prehispánicas, pero de manera universal contribuimos a la transformación del espacio, es decir también a su valorización.

Por eso es que el trabajo arqueológico, con frecuencia, solo permite reconstruir la morfología material es decir cómo se produjo un espacio, al excavar las referencias materiales que han perdurado al paso del tiempo. Solo en cierta circunstancas puede reconstruir la producción del espacio exhumado desde las prácticas de la vida cotidiana y de su simbólica, por lo que se mantienen y se seguirán manteniendo sendas interrogantes sobre la manera cómo se usaban los espacios y el sentido mismo de la producción de estos.

\section{Conclusiones preliminares}

En este recorrido sobre la producción del espacio, hemos transitado desde la versión clásica de cuño marxista y materialista de la producción del espacio a otras de cuño subjetivista. Debe subrayarse que los aportes teóricos de Soja revalorizan también algunas dimensiones de la concepción lefebvriana del espacio: El "Tercer Espacio" como forma de integrar el espacio percibido (la materialidad a la mano) y el espacio concebido (por la subjetividad, la imagnación, etc.) permite escapar a la dialéctica tradicional entre materialidad y subjetividad y recorrer el conocimiento 


\section{La producción del espacio urbano: Entre materialidad y subjetividad}

del espacio de manera distinta (Soja, 2010, en Benach y Albet: 181-209; Soja, 1996)[2]

La dimensión material no carece de interés, pero el enfoque que se ciñe solamente en ella está marcado por su intensa comunión con el relato paratáctico del espacio. Por ende, demuestra que participa de la reificación de los procesos sociales, sino también de la reificación de los mismos individuos que realizan el proceso de manufacturar la ciudad o el espacio en general. Esta limitación del análisis tradicional entra en notoria contradicción con los pasos dados por las ciencias sociales hacia una visión mucho más holística de los procesos sociales.

Por ello, en la segunda parte del texto, se intentó justificar la necesidad de un análisis de las dimensiones subjetivas de la producción del espacio: este enfoque descansa sobre el reconocimiento de que cualquier proceso de producción del espacio es a la vez individual y colectivo. Podemos recordar a este respecto, esta reflexión de David Le Breton cuando señala que "...el hombre de las sociedades holistas conoce una cierta labilidad en un orden del mundo donde todo es mutuamente interrelacionado, un mundo que nunca es dado de una vez por todas. La persona no es entonces formado de los mismos elementos constituyentes, no es delimitada de la misma manera en su relación del mundo" (Le Breton, 2008: 38)[3].

En este contexto, la producción del espacio no es un proceso fijo, firmemente instituido en un mundo sin fallas.
Es por el contrario un proceso marcado por múltiples formas de subjetividad, desde los afectos, los imaginarios y la simbólica, entre otras. También, en ese tenor, debemos considerar a la apropiación del espacio como un momento más de la producción o más directamente, como una forma misma de la producción del espacio.

De tal forma, cualquier sujeto adquiere relevancia porque sus prácticas socioespaciales son consideradas como un proceso de producción; él no es un simple ocupante, un usuario, es un ser dotado de un poder, el de participar en la producción colectiva del espacio.

Este hecho que con frecuencia se suele ignorar, se impone con fuerza cuando la producción del espacio se realiza por medio de marchas o barricadas, como

[2] La obra de Edward Soja se ubica en una corriente neomarxista que renueva radicalmente el pensamiento marxista tradicional sobre la ciudad. Pero a la vez, recupera los textos fundadores, particularmente la producción del Espacio de Lefebvre, un texto donde se pueden encontrar ya muchos aportes sobre una definición del espacio que rebase su materialidad, pero que fue difícilmente aceptado en su tiempo. Por ejemplo Manuel Castells manifiesta que hay que evidenciar la liga ideológica entre la problemática del espacio y la reproducción de la fuerza ed trabajo, como califica al análisis de la cotidianeidad en Lefebvre (Castells, 1972: 115)

[3] El concepto de "labilidad" como inestabilidad, falta de firmeza en las decisiones, es también bastante usado por autores como Michel Maffesoli para caracterizar la actitud del hombre posmoderno (Maffesoli, 2009), contraria a la situación del "Individuo occidental [que] es un hombre garantizado, dado, puesto con firmeza frente a [...] mundo" (Le Breton, 2008: 38) 


\section{La producción del espacio urbano: Entre materialidad y subjetividad}

las que estudió Benjamin, como forma de tomar el espacio, de producir para un grupo y en contra de otro. Es así que en tales casos, esa producción del espacio puede adquirir un carácter revolucionario como lo podemos observar en el quehacer de ciertos movimientos sociales actuales. Pero esto es otro tema que rebasa el horizonte que se fijó para este trabajo, aunque merezca la atención de las ciencias sociales.

\section{Bibliografía}

Adorno, T. (2004). Minima Moralia, reflexiones desde la vida dañada. Madrid: Akal.

Benjamin, W. (2005). El libro de los pasajes. Madrid: Akal.

Buck-Morss, S. (1995). Dialéctica de la Mirada, Walter Benjamin, y el proyecto de los Pasajes. Madrid: Visor, colección "La Balsa de la Medusa", Nº79.

Castells, Manuel (2004). La cuestión urbana. Madrid: Siglo XXI de España.

Hannerz, U. (1986). Exploración de la ciudad, hacia una antropología urbana. México: Fondo de Cultura Económica.

Hiernaux, D. (2007). Los imaginarios urbanos: de la teoría a los aterrizajes en los estudios urbanos. Eure (Vol. XXXIII, N ${ }^{\circ}$ 99), pp. 17-30. Santiago de Chile, agosto.

Honneth, A. (2005). La réification, petit traité de Théorie Critique. Paris: NRF essais.

Le Breton, D. (2008). Anthropologie du corps et modernité. París: Presses Universitaires de France, collection Quadrige-essais débats, 5a edición totalmente revisada.
Lefebvre, H. (1974). La production de l'espace. Paris: Anthropos.

Lefebvre, H. (2013). La producción del espacio. Madrid: Capitan Swing.

Lindón, A. (2007). Los imaginarios urbanos y el constructivismo geográfico: los hologramas espaciales. Revista Eure (Vol. XXXIII, No 99), pp. 31-46. Santiago de Chile, agosto.

Lindón, A. (2010). Los giros teóricos: texto y contexto en: A. Lindón, A. y D. Hiernaux (Eds). Los giros de la geografía humana, desafíos y horizontes. Bar celon a: A n th rop o s y U A M Iztapalapa, pp. 23-41.

Lindón, A. y D. Hiernaux (directores, 2010). Los giros de la geografía humana, desafíos y horizontes. Barcelona: Anthropos y UAM Iztapalapa.

Lindón, A. y D. Hiernaux (directores, 2012). Geografías de lo imaginario. Barcelona: Anthropos y Universidad Autónoma Metropolitana Iztapalapa.

Lukács, G. (1923). "Reificación y conciencia de clase", disponible en inglés e $n \quad$ i $n \quad t \quad e r \quad n \quad e r$; U R L : http://www.marxists.org/ archive/lukacs/works/history/hcc05.htm. consultado el 18 de abril 2015.

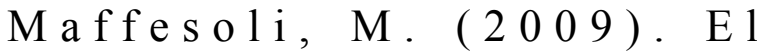
reencantamiento del mundo, una ética para nuestro tiempo. Barcelona: Dedales.

Mejia, P. (2012). Los reggaetoneros y San Judas Tadeo: espacialidad e identidad juvenil a partir de la devoción, el cuerpo y la música. Universidad Autónoma Metropolitana Unidad Iztapalapa, Tesis para la obtención la Licenciatura en Geografía Humana, dirigida por Daniel Hiernaux. 


\section{Bibliografía}

Mejía, P. (2012). Los reggaetoneros y San Judas Tadeo: espacialidad e identidad juvenil a partir de la devoción, el cuerpo y la música. Universidad Autónoma Metropolitana Unidad Iztapalapa, Tesis para la obtención la Licenciatura en Geografía Humana, dirigida por Daniel Hiernaux.

Navarro, P. (1994). El holograma social: una ontología de la socialidad humana. Madrid: Siglo XXI.

Pradilla, E. (1984). Contribución a la crítica de la 'teoría urbana. Del 'espacio' a la 'crisis urbana'. México: Universidad Autónoma Metropolitana Xochimilco.

Soja, E. (2010). Tercer espacio: extendiendo el alcance de la imaginación geográfica. En: Benach, N. y A. Albet (comp.). Edward Soja: La perspectiva posmoderna de un geógrafo radical. Barcelona: Icaria.

Soja, E. (1996). Thirdspace. Journeys to Los Angeles and Other Real and Imagined Spaces. Cambridge: Blackwell.

Turco, A. (2010). Figuras narrativas de la geografía humana. En: A. Lindón y D. Hiernaux (directores). Los giros de la geografía humana, desafíos y horizontes. Barcelona: Anthropos y UAM Iztapalapa, pp. 91-119. 